\title{
HIGH-ORDER COLLOCATION METHODS FOR DIFFERENTIAL EQUATIONS WITH RANDOM INPUTS*
}

\author{
DONGBIN XIU ${ }^{\dagger}$ AND JAN S. HESTHAVEN ${ }^{\ddagger}$
}

\begin{abstract}
Recently there has been a growing interest in designing efficient methods for the solution of ordinary/partial differential equations with random inputs. To this end, stochastic Galerkin methods appear to be superior to other nonsampling methods and, in many cases, to several sampling methods. However, when the governing equations take complicated forms, numerical implementations of stochastic Galerkin methods can become nontrivial and care is needed to design robust and efficient solvers for the resulting equations. On the other hand, the traditional sampling methods, e.g., Monte Carlo methods, are straightforward to implement, but they do not offer convergence as fast as stochastic Galerkin methods. In this paper, a high-order stochastic collocation approach is proposed. Similar to stochastic Galerkin methods, the collocation methods take advantage of an assumption of smoothness of the solution in random space to achieve fast convergence. However, the numerical implementation of stochastic collocation is trivial, as it requires only repetitive runs of an existing deterministic solver, similar to Monte Carlo methods. The computational cost of the collocation methods depends on the choice of the collocation points, and we present several feasible constructions. One particular choice, based on sparse grids, depends weakly on the dimensionality of the random space and is more suitable for highly accurate computations of practical applications with large dimensional random inputs. Numerical examples are presented to demonstrate the accuracy and efficiency of the stochastic collocation methods.
\end{abstract} cation

Key words. collocation methods, stochastic inputs, differential equations, uncertainty quantifi-

AMS subject classifications. $65 \mathrm{C} 20,65 \mathrm{C} 30$

DOI. $10.1137 / 040615201$

1. Introduction. This paper is devoted to numerical solutions of differential equations with random inputs. The source of random inputs includes uncertainty in system parameters, boundary/initial conditions, etc. Such kinds of uncertainty are ubiquitous in engineering applications and are often modeled as random processes. The classical approach is to model the random inputs as some idealized processes which typically are white noises, e.g., Wiener processes, Poisson processes, etc., and has led to elegant mathematical analysis and numerical methods by using stochastic calculus, e.g., Ito or Strantonovich calculus. The resulting differential equations are termed "stochastic ordinary/partial differential equations" (SODE/SPDE) (see, for example, $[18,25,28,43])$. Recently, there has been a growing interest in studying problems with more correlated random inputs ("colored noises") and, in case of random parameters, fully correlated random processes, i.e., random variables. In this context, the classical stochastic calculus does not readily apply and other approaches are required.

*Received by the editors September 16, 2004; accepted for publication (in revised form) August 5, 2005; published electronically December 30, 2005.

http://www.siam.org/journals/sisc/27-3/61520.html

${ }^{\dagger}$ Department of Mathematics, Purdue University, West Lafayette, IN 47907 (dxiu@math.purdue. edu). The work of this author was partly supported by AFOSR contract F49620-01-1-0035.

${ }^{\ddagger}$ Division of Applied Mathematics, Brown University, Providence, RI 02912 (jansh@dam.brown. edu). The work of this author was partly supported by NSF Career Award DMS-0132967, by AFOSR contract FA9550-04-1-0072, and by the Alfred P. Sloan Foundation through a Sloan Research Fellowship. 
The traditional approach for differential equations with random inputs is the Monte Carlo method, which generates ensembles of random realizations for the prescribed random inputs and utilizes repetitive deterministic solvers for each realization. Monte Carlo methods have been applied to many applications and their implementations are straightforward. Although the convergence rate - the ensemble mean of a brute-force Monte Carlo method with $K$ realizations converges asymptotically at a rate $1 / \sqrt{K}$ - is relatively slow, it is (formally) independent of the dimensionality of the random space, i.e., independent of the number of random variables used to characterize the random inputs (e.g., [13]). To accelerate convergence, several techniques have been developed, for example, Latin hypercube sampling, [34, 48], the quasi-Monte Carlo (QMC) method, [14, 39, 40], and the Markov chain Monte Carlo method (MCMC) $[16,22,36]$. These methods can improve the efficiency of the bruteforce Monte Carlo method. However, additional restrictions are imposed based on their specific designs and their applicability is limited.

Another class of methods is the nonsampling methods, where no repetitive deterministic solvers are employed. Methods along this line of approach include perturbation methods [27] and second-moment analysis [31, 32]. These methods do not result in convergent series of expansion and their applicability is restricted to systems with relatively small random inputs and outputs. Such a condition is difficult to satisfy, especially for nonlinear problems where small random inputs may induce large random outputs (e.g., [56]). Stochastic Galerkin methods, whose convergence can be established for problems with relatively large magnitude of random inputs/outputs, have been developed. The stochastic Galerkin methods are generalizations of the Wiener-Hermite polynomial chaos expansion, which was developed in [51] and has been applied to various problems in mechanics [21]. The generalizations, also called generalized polynomial chaos, employ non-Hermite polynomials to improve efficiency for a wider class of problems and include global polynomial expansions based on hypergeometrical polynomials [53, 52, 54], piecewise polynomial expansions [2, 12], and wavelet basis expansions $[29,30]$. In general, these methods exhibit fast convergence rates with increasing order of the expansions, provided that solutions are sufficiently smooth in the random space. However, the resulting set of deterministic equations is often coupled and care is needed to design efficient and robust solver. (See [52, 55] for examples of diffusion equations.) The form of the resulting equations can become very complicated if the underlying differential equations have nontrivial and nonlinear forms. (See $[7,37,57]$ for examples of hyperbolic systems.)

In this paper, we propose a class of stochastic collocation methods that combines the strength of Monte Carlo methods and stochastic Galerkin methods. By taking advantage of the existing theory on multivariate polynomial interpolations (e.g., $[5,6]$ ), the stochastic collocation methods achieve fast convergence when the solutions possess sufficient smoothness in random space, similar to stochastic Galerkin methods. On the other hand, the implementations of stochastic collocation methods are straightforward as they only require solutions of the corresponding deterministic problems at each interpolation point, similar to Monte Carlo methods at each sampling point. Such properties make stochastic collocation methods good alternatives to Monte Carlo methods and stochastic Galerkin methods. The core issue is the construction of the set of interpolation points, and it is nontrivial in multidimensional random spaces. For such a cases, point sets based on tensor products of one-dimensional quadrature points $[37,53]$ are not suitable, as the number of points grows too fast with increasing dimensions. Therefore, we investigate and propose two other choices. One is based on Stroud's cubature points [49] and the other on sparse grids from the Smolyak 
algorithm [46]. The former approach is of relatively low-order accuracy but results in highly efficient algorithms, especially for problems with large dimensional random inputs. The latter offers true high-order accuracy with convergence rate depending weakly on dimensionality. Both methods are more efficient than brute-force Monte Carlo methods in a large range of random dimensionality. Compared to stochastic Galerkin methods, the collocation methods generally result in a larger number of equations than a typical Galerkin method; however, these equations are easier to solve as they are completely decoupled and require only repetitive runs of a deterministic solver. Such a property makes the collocation methods more attractive for problems with complicated governing equations.

This paper is organized as follows. In section 2 we present the mathematical framework of stochastic differential equations - the functional spaces and the strong and weak forms are formulated in section 2.1 and we briefly review the Monte Carlo methods and stochastic Galerkin methods in section 2.2. The stochastic collocation methods are discussed in section 3, where the construction of collocation points is discussed in detail. More technical details and numerical examples are presented in sections 4 and 5 , and we conclude the paper in section 6 .

2. Random differential equations. Let $(\Omega, \mathcal{A}, \mathcal{P})$ be a complete probability space, where $\Omega$ is the event space, $\mathcal{A} \subset 2^{\Omega}$ the $\sigma$-algebra, and $\mathcal{P}$ the probability measure. Consider a $d$-dimensional bounded domain $D \subset \mathbb{R}^{d}(d=1,2,3)$ with boundary $\partial D$, and we study the following problem: find a stochastic function, $u \equiv$ $u(\omega, x): \Omega \times \bar{D} \rightarrow \mathbb{R}$, such that for $\mathcal{P}$-almost everywhere $\omega \in \Omega$, the following equation holds:

$$
\mathcal{L}(\omega, x ; u)=f(\omega, x), \quad x \in D,
$$

subject to the boundary condition

$$
\mathcal{B}(\omega, x ; u)=g(\omega, x), \quad x \in \partial D,
$$

where $x=\left(x_{1}, \ldots, x_{d}\right)$ are the coordinates in $\mathbb{R}^{d}, \mathcal{L}$ is a (linear/nonlinear) differential operator, and $\mathcal{B}$ is a boundary operator. The operator $\mathcal{B}$ can take various forms on different boundary segments, e.g., $\mathcal{B} \equiv \mathbb{I}$ on Dirichlet segments (where $\mathbb{I}$ is the identity operator) and $\mathcal{B} \equiv n \cdot \nabla$ on Neumann segments (where $n$ is the outward unit normal vector). In the most general settings, the operators $\mathcal{L}$ and $\mathcal{B}$, as well as the driving terms $f$ and $g$, can all have random components. Finally, we assume that the boundary $\partial D$ is sufficiently regular and the driving terms $f$ and $g$ are properly posed, such that (2.1)-(2.2) is well-posed $\mathcal{P}$-a.e. $\omega \in \Omega$. (With a slight abuse of notation, (2.1)-(2.2) can incorporate time-dependent problems, if we consider $D$ to be in an $\left(\mathbb{R}^{d+1}\right)$-dimensional space, with the extra dimension designated to time.)

2.1. Strong and weak forms. To solve (2.1)-(2.2) numerically, we need to reduce the infinite-dimensional probability space to a finite-dimensional space. This can be accomplished by characterizing the probability space by a finite number of random variables. Such a procedure, termed the "finite-dimensional noise assumption" in [2], is often achieved via a certain type of decomposition which can approximate the target random process with desired accuracy. One of the choices is the Karhunen-Loève type expansion [33], which is based on the spectral decomposition of the covariance function of the input random process. Following a decomposition and assuming that the random inputs can be characterized by $N$ random variables, we can rewrite the 
random inputs in abstract form, e.g.,

$$
\mathcal{L}(\omega, x ; u)=\mathcal{L}\left(Y^{1}(\omega), \ldots, Y^{N}(\omega), x ; u\right), \quad f(\omega, x)=f\left(Y^{1}(\omega), \ldots, Y^{N}(\omega), x\right),
$$

where $\left\{Y^{i}\right\}_{i=1}^{N}$ are real random variables with zero mean value and unit variance. Hence, following the Doob-Dynkin lemma [43], the solution of (2.1)-(2.2) can be described by the same set of random variables $\left\{Y^{i}(\omega)\right\}_{i=1}^{N}$, i.e.,

$$
u(\omega, x)=u\left(Y^{1}(\omega), \ldots, Y^{N}(\omega), x\right) .
$$

By characterizing the probability space with $N$ random variables, we have effectively reduced the infinite-dimensional probability space to a $N$-dimensional space.

From a mathematical and computational point of view, it is most convenient to further assume that these random variables are mutually independent, and subsequently, we can define the corresponding Hilbert functional spaces via simple tensor product rules. However, such an assumption could potentially introduce more errors in approximating the input random processes (in addition to the errors induced by truncating the infinite series during a decomposition procedure such as the KarhunenLoève expansion). For example, for non-Gaussian processes, the Karhunen-Loève expansion yields a set of uncorrelated random variables that are not necessarily mutually independent. To transform them into independent random variables, a (nonlinear) transformation is needed and its numerical construction can be nontrivial. In fact, a mathematically rigorous transformation exists by using merely the definition of joint cumulative density function (CDF) [44]. However, such a transformation is of little practical use as it requires the knowledge of all the joint CDFs of the underlying random process.

In this paper, we take the customary approach (see, for example, $[2,30,52]$ ) of assuming that the random inputs are already characterized by a set of mutually independent random variables with satisfactory accuracy. We emphasize that how to (numerically) achieve this, i.e., to represent continuous non-Gaussian random inputs via the Karhunen-Loève decomposition or any other representation and to ensure that the random variables involved are independent, falls into the topic of numerical representation of non-Gaussian processes. It remains an active research area (e.g., $[23,45,58])$ and is beyond the scope of this paper. We also remark that it is possible to construct multidimensional functional spaces based on finite number of dependent random variables [47]. However, such a construction does not, in its current form, allow straightforward numerical implementations.

Let us now assume that $\left\{Y^{i}\right\}_{i=1}^{N}$ are independent random variables with probability density functions $\rho_{i}: \Gamma^{i} \rightarrow \mathbb{R}^{+}$and their images $\Gamma^{i} \equiv Y^{i}(\Omega)$ are bounded intervals in $\mathbb{R}$ for $i=1, \ldots, N$. Then

$$
\rho(y)=\prod_{i=1}^{N} \rho_{i}\left(Y^{i}\right) \quad \forall y \in \Gamma
$$

is the joint probability density of $y=\left(Y^{1}, \ldots, Y^{N}\right)$ with the support

$$
\Gamma \equiv \prod_{i=1}^{N} \Gamma^{i} \subset \mathbb{R}^{N} .
$$


This allows us to rewrite (2.1)-(2.2) as an $(N+d)$-dimensional differential equation in the strong form

$$
\mathcal{L}(y, x ; u)=f(y, x), \quad(y, x) \in \Gamma \times D,
$$

subject to the boundary condition

$$
\mathcal{B}(y, x ; u)=g(y, x), \quad(y, x) \in \Gamma \times \partial D,
$$

where $N$ is the dimensionality of the random space $\Gamma$ and $d$ is the dimensionality of the physical space $D$.

Similar to the weak formulations in deterministic problems, we often seek to solve (2.7)-(2.8) in a weak form. For example, we can define a (finite-dimensional) subspace $V_{\Gamma} \subset L_{\rho}^{2}(\Gamma)$, the space of all square integrable function in $\Gamma$ with respect to the measure $\rho(y) d y$, and seek $u_{V}(y, x) \in V_{\Gamma}(y)$, such that

$$
\begin{aligned}
& \int_{\Gamma} \rho(y) \mathcal{L}\left(y, x ; u_{V}\right) v(y) d y=\int_{\Gamma} \rho(y) f(y, x) v(y) d y \quad \forall v(y) \in V_{\Gamma}, x \in D, \\
& \int_{\Gamma} \rho(y) \mathcal{B}\left(y, x, u_{V}\right) v(y) d y=\int_{\Gamma} \rho(y) g(y, x) v(y) d y \quad \forall v(y) \in V_{\Gamma}, x \in \partial D .
\end{aligned}
$$

The resulting problem (2.9)-(2.10) thus becomes a deterministic problem in the physical domain $D$ and can be solved by a standard discretization technique, e.g., finite elements, finite volume.

2.2. Existing numerical methods. Several numerical methods can be applied to problem (2.7)-(2.8), or (2.9)-(2.10). Here we briefly review two of the more popular methods - Monte Carlo methods and stochastic Galerkin methods - whose strengths complement each other.

2.2.1. Monte Carlo methods. Monte Carlo simulation is one of the most developed methods for solving stochastic differential equations. Although the convergence rate is relatively slow - a typical Monte Carlo simulation consisting of $K$ realizations converges asymptotically as $1 / \sqrt{K}[13]$ - it is independent of the number of random variables $\left\{Y^{i}(\omega)\right\}_{i=1}^{N}$. The procedure of applying a Monte Carlo method to problem (2.7)-(2.8) takes the following steps:

1. For a prescribed number of realizations $K$, generate independent and identically distributed (i.i.d.) random variables $\left\{Y_{j}^{i}\right\}_{i=1}^{N} \equiv\left\{Y^{i}\left(\omega_{j}\right)\right\}_{i=1}^{N}$, for $j=1, \ldots, K$

2. For each $j=1, \ldots, K$, solve a deterministic problem (2.7)-(2.8) with $y_{j}=$ $\left(Y_{j}^{1}, \ldots, Y_{j}^{N}\right)$ and obtain solution $u_{j} \equiv u\left(y_{j}, x\right)$, i.e., solve $\mathcal{L}\left(y_{j}, x ; u_{j}\right)=$ $f\left(y_{j}, x\right)$ in $D$, subject to $\mathcal{B}\left(y_{j}, x ; u_{j}\right)=g\left(y_{j}, x\right)$ on $\partial D$;

3. Postprocess the results to evaluate the solution statistics, e.g., $\langle u\rangle \equiv \mathbb{E}[u]=$ $\frac{1}{M} \sum_{j=1}^{M} u_{j}$.

Here $\mathbb{E}[u]=\int_{\Gamma} \rho(y) u(y) d y$ is the expectation. Note that for each realization $j=$ $1, \ldots, K$, step 2 is a deterministic problem and can be solved by any suitable scheme.

2.2.2. Stochastic Galerkin methods. Stochastic Galerkin methods deal with the weak form (2.9)-(2.10), and they offer fast convergence when the solution is sufficiently smooth in the random space. Forms of stochastic Galerkin methods differ in the construction of the subspace $V_{\Gamma}$ in (2.9). Here we briefly review the construction 
based on global orthogonal polynomial expansions. Let us define one-dimensional subspaces for $L_{\rho_{i}}^{2}\left(\Gamma^{i}\right)$,

$$
W^{i, p_{i}} \equiv\left\{v: \Gamma^{i} \rightarrow \mathbb{R}: v \in \operatorname{span}\left\{\phi_{m}\left(Y^{i}\right)\right\}_{m=0}^{p_{i}}\right\}, \quad i=1, \ldots, N,
$$

where $\left\{\phi_{m}\left(Y^{i}\right)\right\}$ are a set of orthogonal polynomials satisfying the orthogonality conditions

$$
\int_{\Gamma_{i}} \rho_{i}\left(Y^{i}\right) \phi_{m}\left(Y^{i}\right) \phi_{n}\left(Y^{i}\right) d Y^{i}=h_{m}^{2} \delta_{m n}
$$

with normalization factors $h_{m}^{2}=\int_{\Gamma_{i}} \rho_{i} \phi_{m}^{2} d Y^{i}$. The type of the orthogonal polynomials $\left\{\phi_{m}\left(Y^{i}\right)\right\}$ in (2.12) is determined by $\rho_{i}\left(Y^{i}\right)$, the probability density function of $Y^{i}(\omega)$, for $i=1, \ldots, N$. For example, uniform distributions are better approximated by Legendre polynomials, and Hermite polynomials are more appropriate for Gaussian distributions. (See $[53,52]$ for a detailed list of correspondences.) We remark that such a correspondence is not mandatory, so long as $\left\{\phi_{m}\left(Y^{i}\right)\right\}$ forms a complete basis.

The corresponding $N$-dimensional subspace $V_{\Gamma}$ for $\Gamma$ can be defined as

$$
W_{N}^{p} \equiv \bigotimes_{|\mathbf{p}| \leq p} W^{i, p_{i}}
$$

where the tensor product is over all possible combinations of the multi-index $\mathbf{p}=$ $\left(p_{1}, \ldots, p_{N}\right) \in \mathbb{N}_{0}^{N}$ satisfying $|\mathbf{p}|=\sum_{i=1}^{N} p_{i} \leq p$. Thus, $W_{N}^{p}$ is the space of $N$ variate orthogonal polynomials of total degree at most $p$, and the number of basis functions in (2.13) is $\operatorname{dim}\left(W_{N}^{p}\right)=\left(\begin{array}{c}N+p \\ N\end{array}\right)$. Hereafter, we will refer to $W_{N}^{p}$ as a complete polynomial space. Such spaces are often employed in the (generalized) polynomial chaos expansions $[21,52,54]$.

Another construction of $V_{\Gamma}$ is based on the tensor products of the one-dimensional polynomial spaces with fixed highest polynomial orders in all dimensions, i.e.,

$$
Z_{N}^{p}=\bigotimes_{i=1}^{N} W^{i, p_{i}}, \quad \max _{i} p_{i}=p .
$$

Such a space will be denoted as tensor polynomial space hereafter. They are employed in $[2,15]$, and the number of basis functions in $Z_{N}^{p}$ is $\operatorname{dim}\left(Z_{N}^{p}\right)=(p+1)^{N}$.

If the same one-dimensional subspaces (2.11) are employed, then $W_{N}^{p} \subset Z_{N}^{p}$. The numbers of basis functions in both spaces depend on the number of dimensions $N$ and the polynomial orders $p$, and they grow fast with increasing $N$ and $p$. In particular, the total number of terms can grow exponentially fast with increasing order $p$ when $N \gg 1$ (curse-of-dimensionality). We also remark that when $N \gg 1$ and $p$ is moderate (e.g. $p \leq 10), \operatorname{dim}\left(W_{N}^{p}\right) \ll \operatorname{dim}\left(Z_{N}^{p}\right)$.

For low to moderate value of $N$, stochastic Galerkin methods are preferred because their high accuracy and fast convergence; for large value of $N \gg 1$, the rapidly growing number of basis functions effectively reduces its efficiency. In this case, the Monte Carlo method is preferred. However, if a highly accurate stochastic solution is desired, then stochastic Galerkin methods can be more efficient even for relatively large value of $N$ (e.g., [2]). 
3. Stochastic collocation methods. In this section, we present high-order collocation methods for (2.1)-(2.2). The objective is to combine the strength of the two existing approaches: the high resolution of stochastic Galerkin methods resulting from polynomial approximations in random spaces, and the ease of implementation of Monte Carlo methods by sampling at discrete points in random spaces.

3.1. Formulation. The construction of high-order stochastic collocation methods is based on polynomial interpolations in the multidimensional random space. Let us denote by $y=\left(Y^{1}, \ldots, Y^{N}\right)$ any point in the random space $\Gamma \subset \mathbb{R}^{N}$, by $\Pi_{N}$ the space of all $N$-variate polynomials with real coefficients, and by $\Pi_{N}^{p}$ the subspace of polynomials of total degree at most $p$. (Note that the space $W_{N}^{p}$ defined in (2.13) is a subset of $\Pi_{N}^{p}$.) When derivatives are not interpolated, the Lagrange interpolation problem can be stated in the following form.

DeFINITION 3.1 (Lagrange interpolation). Given a finite number of distinct points $y_{1}, \ldots, y_{M}$, some real constants $b_{1}, \ldots, b_{M}$, and a subspace $V_{I} \in \Pi_{N}$, find a polynomial $l \in V_{I}$ such that

$$
l\left(y_{j}\right)=b_{j}, \quad j=1, \ldots, M .
$$

The points $y_{1}, \ldots, y_{M}$ are called interpolation nodes, and $V_{I}$ is the interpolation space.

DEFINITION 3.2 (poisedness). The Lagrange interpolation problem in Definition 3.1 , for the points $y_{1}, \ldots, y_{M} \in \mathbb{R}^{N}$, is called poised in $V_{I}$ if, for any given data $b_{1}, \ldots, b_{M} \in \mathbb{R}$, there exists a function $f \in V_{I}$ such that $f\left(y_{j}\right)=b_{j}, j=1, \ldots, M$. When the Lagrange interpolation problem for any $M$ distinct points in $\mathbb{R}^{N}$ is poised in $V_{I}$, then $V_{I}$ is called a Haar space of order $M$.

There is a well-developed and extensive classical theory of univariate Lagrange polynomial interpolation. However, the multivariate case is more difficult. In particular, the Haar spaces exist in abundance for $N=1$ but not for $N>1$. In fact, in this case there are no Haar spaces of dimension greater than one - the so-called loss-of-Haar. (For more details and the refinement of this statement, see [11,35].) Thus, research has focused on the selection of points $\left\{y_{i}\right\}_{i=1}^{M}$ such that one achieves a good approximation by a Lagrange interpolation.

Let $\Theta_{N}=\left\{y_{i}\right\}_{i=1}^{M} \in \Gamma$ be a set of (prescribed) nodes such that the Lagrange interpolation (3.1) in the $N$-dimensional random space $\Gamma$ is poised in a corresponding interpolation space $V_{I}$. (For sufficient conditions on the distribution of the nodes to ensure a unique interpolation, see [8].) Subsequently, Lagrange interpolation of a smooth function, $f: \mathbb{R}^{N} \rightarrow \mathbb{R}$, can now be viewed as follows: find a polynomial $\mathcal{I}(f) \in V_{I}$ such that $\mathcal{I}(f)\left(y_{i}\right)=f\left(y_{i}\right), \forall i=1, \ldots, M$.

The polynomial approximation $\mathcal{I}(f)$ can be expressed by using the Lagrange interpolation polynomials, i.e.,

$$
\mathcal{I}(f)(y)=\sum_{k=1}^{M} f\left(y_{k}\right) L_{k}(y),
$$

where

$$
L_{i}(y) \in V_{I}, \quad L_{i}\left(y_{j}\right)=\delta_{i j}, \quad 1 \leq i, j \leq M,
$$

are the Lagrange polynomials.

We equip the space $V_{I}$ with the supremum-norm $\|\cdot\|$ and introduce

$$
\|\mathcal{I}\|=\sup _{f \neq 0} \frac{\|\mathcal{I} f\|_{\infty}}{\|f\|_{\infty}}
$$


The Lebesgue theorem states that the interpolation error is uniformly bounded as

$$
\left\|f(y)-f^{*}(y)\right\|_{\infty} \leq\|f(y)-\mathcal{I} f(y)\|_{\infty} \leq(1+\Lambda)\left\|f(y)-f^{*}(y)\right\|_{\infty},
$$

where $f^{*}(y)$ is the best approximating polynomial, and $\Lambda=\|\mathcal{I}\|=\max _{y \in \Gamma} \sum_{k=1}^{K}\left|L_{i}(y)\right|$ is the Lebesgue constant. We remark that the determination of $f^{*}(y)$ is an unsolved problem for arbitrary $(N>1)$-dimensional spaces, and the estimation of the Lebesgue constant, which depends on the particular choice of nodal sets $\Theta_{N}$, is a nontrivial task even for $N=1$.

By denoting

$$
\hat{u}(y) \equiv \mathcal{I} u(y)=\sum_{k=1}^{M} u\left(y_{k}\right) L_{k}(y),
$$

the collocation procedure to solve the stochastic elliptic equation (2.7) is

$$
\left.R(\hat{u}(y))\right|_{y_{k}}=0 \quad \forall k=1, \ldots, M,
$$

where $R(u)=\mathcal{L}(u)-f$ is the residual of $(2.7)$. By using the property of Lagrange interpolation (3.3), we immediately obtain: for $k=1, \ldots, M$,

$$
\mathcal{L}\left(y_{k}, x ; u\right)=f\left(y_{k}, x\right), \quad x \in D,
$$

with boundary condition

$$
\mathcal{B}\left(y_{k}, x ; u\right)=g\left(y_{k}, x\right), \quad x \in \partial D .
$$

Thus, the stochastic collocation method is equivalent to solving $M$ deterministic problems (3.8)-(3.9), the deterministic counterpart of problem (2.7)-(2.8), at each nodal point $y_{k}, k=1, \ldots, M$ in a given nodal set $\Theta_{N}$. Note that the problem (3.8)-(3.9) for each $k$ is naturally decoupled, and existing deterministic solvers can be readily applied. This is in direct contrast to the stochastic Galerkin approaches, where the resulting expanded equations are, in general, coupled.

Once the numerical solutions of (3.8)-(3.9) are obtained at all collocation points, the statistics of the random solution can be evaluated, e.g.,

$$
\mathbb{E}(\hat{u})(x)=\sum_{k=1}^{M} u\left(y_{k}, x\right) \int_{\Gamma} L_{k}(y) \rho(y) d y .
$$

The evaluations of such expectations require explicit knowledge of the Lagrange interpolation polynomials $\left\{L_{k}(y)\right\}$. For a given nodal set $\Theta_{N}$, the polynomials can be determined numerically by inverting a Vandermonde-type matrix. (The matrix is invertible under the assumption that the Lagrange interpolation is poised on $\Theta_{N}$.) In multivariate cases, such a procedure can be cumbersome, but can be accomplished once and for all at the preprocessing stage.

An alternative is to choose the set $\Theta_{N}$ to be a cubature point set. Recall that a cubature rule approximates an integral by

$$
\int_{\Gamma} f(y) d y \simeq \sum_{i=1}^{M} f\left(y_{i}\right) w_{i}
$$


where $\left\{w_{i}\right\}$ are the weights. It is called "of order $s$ " if the approximation is exact for all $f \in \Pi_{N}^{r}, r \leq s$, and not exact for at least one $f \in \Pi_{N}^{s+1}$. When we choose the interpolation point set $\Theta_{N}$ to be the same as a cubature point set, the evaluation of (3.10) is reduced to

$$
\mathbb{E}(\hat{u})(x)=\sum_{k=1}^{M} u\left(y_{k}, x\right) w_{k}
$$

It should be noted that the use of cubature formula to evaluate the solution statistics will introduce extra errors whenever the integrals defining these statistics, e.g., (3.10) for mean solution, cannot be evaluated exactly by an $s$-order cubature.

Construction of cubature formulas has long been an active and challenging research area, and we refer interested readers to $[10,9]$ for extensive reviews.

3.2. Choices of collocation points. The computational complexity of the stochastic collocation methods (3.8)-(3.9) is $M$ times that of a deterministic problem, where $M$ is the total number of collocation points. Thus, we need to choose a nodal set $\Theta_{N}$ with fewest possible number of points under a prescribed accuracy requirement. In this section, we present several choices of such collocation points. The main focus here is on multidimensional random spaces, i.e., $\Gamma \subset \mathbb{R}^{N}$ with $N>1$. Without loss of generality, we assume that the bounded support of the random variables $Y^{i}$ is $\Gamma^{i}=[-1,1]$ for $i=1, \ldots, N$ and subsequently the bounded random space $\Gamma$ is a $N$ hypercube, i.e., $\Gamma=[-1,1]^{N}$. (Note that random variables with bounded support in $[a, b]$ can always be mapped to $[-1,1]$, and any domain $\Gamma^{*}=\prod_{i=1}^{N}\left[a^{i}, b^{i}\right]$ to $[-1,1]^{N}$.)

3.2.1. Tensor products of one-dimensional nodal sets. A natural choice of the nodal set is the tensor product of one-dimensional sets. In interpolating smooth functions $f:[-1,1]^{N} \rightarrow \mathbb{R}$, much is known about good interpolation formulas for $N=1$, i.e., for every direction $i=1, \ldots, N$, we can construct a good one-dimensional interpolation

$$
\mathcal{U}^{i}(f)=\sum_{k=1}^{m_{i}} f\left(Y_{k}^{i}\right) \cdot a_{k}^{i}
$$

based on nodal sets

$$
\Theta_{1}^{i}=\left(Y_{1}^{i}, \ldots, Y_{m_{i}}^{i}\right) \subset[-1,1],
$$

where $a_{k}^{i} \equiv a_{k}\left(Y^{i}\right) \in C([-1,1])$. We assume that a sequence of formulas (3.13) is given. In the multivariate case $N>1$, the tensor product formulas are

$$
\mathcal{I}(f) \equiv\left(\mathcal{U}^{i_{1}} \otimes \cdots \otimes \mathcal{U}^{i_{N}}\right)(f)=\sum_{k_{1}=1}^{m_{i_{1}}} \cdots \sum_{k_{N}=1}^{m_{i_{N}}} f\left(Y_{k_{1}}^{i_{1}}, \ldots, Y_{k_{N}}^{i_{N}}\right) \cdot\left(a_{k_{1}}^{i_{1}} \otimes \cdots \otimes a_{k_{N}}^{i_{N}}\right) .
$$

Clearly, the above product formula needs $M=m_{i_{1}} \cdots m_{i_{N}}$ nodal points. If we choose to use the same interpolating function (3.13) in each dimension with the same number of points, i.e., $m_{i_{1}}=\cdots=m_{i_{N}} \equiv m$, the total number of points is $M=m^{N}$. This number grows quickly in high dimensions $N \gg 1$ - even for a poor approximation with two points $(m=2)$ in each dimension, $M=2^{N} \gg 1$ for $N \gg 1$. 
The tensor product interpolation is easy to construct - the interpolation points and space are obtained by tensor products of the univariate ones. The Lagrange formula are easily extended to this case, as can be found in $[4,24]$. However, because of the rapidly growing number of interpolation nodes in high dimensions, we will not consider this approach extensively in this paper.

3.2.2. Sparse grids. In this section we propose to solve the stochastic collocation problem (3.8)-(3.9) on a sparse grid constructed by the Smolyak algorithm. The Smolyak algorithm is a linear combination of product formulas, and the linear combination is chosen in such a way that an interpolation property for $N=1$ is preserved for $N>1$. Only products with a relatively small number of points are used and the resulting nodal set has significantly less number of nodes compared to the tensor product rule (3.15). Much research has been devoted to the Smolyak algorithm since its introduction in [46]; see, e.g., [3, 41, 42].

Starting with the one-dimensional interpolation formula (3.13), the Smolyak algorithm is given by (see [50])

$$
\mathcal{I}(f) \equiv A(q, N)=\sum_{q-N+1 \leq \mathbf{i} \mid \leq q}(-1)^{q-|\mathbf{i}|_{1}} \cdot\left(\begin{array}{c}
N-1 \\
q-|\mathbf{i}|
\end{array}\right) \cdot\left(\mathcal{U}^{i_{1}} \otimes \cdots \otimes \mathcal{U}^{i_{N}}\right),
$$

where $\mathbf{i}=\left(i_{1}, \ldots, i_{N}\right) \in \mathbb{N}^{N}$. To compute $A(q, N)$, we only need to evaluate function on the "sparse grid"

$$
\Theta_{N} \equiv \mathcal{H}(q, N)=\bigcup_{q-N+1 \leq|\mathbf{i}| \leq q}\left(\Theta_{1}^{i_{1}} \times \cdots \times \Theta_{1}^{i_{N}}\right)
$$

In this paper, we choose to use Smolyak formulas that are based on one-dimensional polynomial interpolation at the extrema of the Chebyshev polynomials. (Other choices, such as the Gauss quadrature points, can be considered as well.) For any choice of $m_{i}>1$, these nodes are given by

$$
Y_{j}^{i}=-\cos \frac{\pi \cdot(j-1)}{m_{i}-1}, \quad j=1 \ldots, m_{i} .
$$

In addition, we define $Y_{1}^{i}=0$ if $m_{i}=1$. The functions $a_{j}^{i}$ in (3.13) are characterized by the requirement that $\mathcal{U}^{i}$ reproduce all polynomials of degree less than $m_{i}$. We also choose $m_{1}=1$ and $m_{i}=2^{i-1}$ for $i>1$. By doing so, the one-dimensional nodal sets $\Theta_{1}^{i}$ are nested, i.e., $\Theta_{1}^{i} \subset \Theta_{1}^{i+1}$, and subsequently $\mathcal{H}(q, N) \subset \mathcal{H}(q+1, N)$.

It has been shown that if we set $q=N+k$, then $A(N+k, N)$ is exact for all polynomials in $\Pi_{N}^{k}$ [42], and the total number of nodes for $N \gg 1$ is [41]

$$
M \equiv \operatorname{dim}(A(N+k, N)) \sim \frac{2^{k}}{k !} N^{k}, \quad k \text { fixed, } N \gg 1 .
$$

On the other hand, $\operatorname{dim}\left(\Pi_{N}^{k}\right)=\left(\begin{array}{c}N+k \\ N\end{array}\right) \sim N^{k} / k$ ! for $N \gg 1$. Thus, $A(N+k, N)$ uses about $2^{k}$ times as many points as the degree-of-freedom of $\Pi_{N}^{k}$. Since this factor is independent of $N$, the algorithm may be regarded as optimal. Formula (3.19) also indicates that the number of nodes from the Smolyak sparse grid is larger than the number of expansion terms of a stochastic Galerkin method in the complete polynomial space $W_{N}^{k}$. Hereafter, we will refer $k$ in $A(N+k, N)$ as the "level" of the Smolyak construction. 

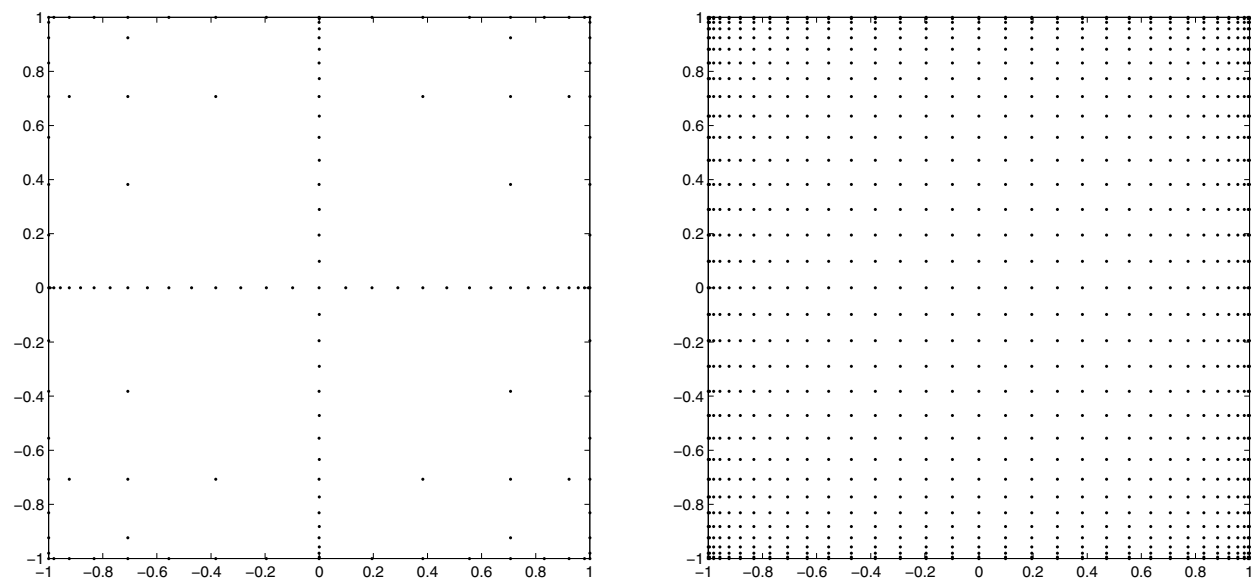

FIG. 3.1. Two-dimensional $(N=2)$ interpolation nodes based on the extrema of Chebyshev polynomials (3.18). Left: sparse grid $\mathcal{H}(N+k, N)$ from Smolyak algorithm, $k=5$. Total number of points is 145. Right: tensor product algorithm (3.15) from the same one-dimensional nodes. Total number of nodes is 1,089 .

TABLE 3.1

The degrees-of-freedom of the Smolyak sparse grid $(\mathcal{H}(N+k, N)=\operatorname{dim}(A(N+k, N)))$, the complete polynomial space $W_{N}^{k}$, and the tensor polynomial space $Z_{N}^{k}$, for various dimensions $N$ and order $k$.

\begin{tabular}{|c|c|c|c|c|}
\hline$N$ & $k$ & $\mathcal{H}(N+k, N)$ & $\operatorname{dim}\left(W_{N}^{k}\right)$ & $\operatorname{dim}\left(Z_{N}^{k}\right)$ \\
\hline 2 & 1 & 5 & 3 & 4 \\
& 2 & 13 & 6 & 9 \\
& 3 & 29 & 10 & 16 \\
& 4 & 65 & 15 & 25 \\
\hline 10 & 1 & 21 & 11 & 1,024 \\
& 2 & 221 & 66 & 59,049 \\
& 3 & 1,581 & 286 & $1,048,576$ \\
\hline 20 & 1 & 41 & 21 & $1,048,576$ \\
& 2 & 841 & 231 & $\approx 3.5 \times 10^{9}$ \\
\hline 50 & 1 & 101 & 51 & $\approx 1.1 \times 10^{16}$ \\
& 2 & 5,101 & 1,326 & $\approx 7.2 \times 10^{23}$ \\
\hline
\end{tabular}

In Figure 3.1, we show the two-dimensional $(N=2)$ interpolation nodes by the sparse grid $\mathcal{H}(N+k, N)$ described above, with $k=5$. For comparison, the twodimensional tensor product grid based on the same one-dimensional nodes are shown on the right of Figure 3.1, and we observe that the sparse grid has significantly fewer nodes.

In Table 3.1 we list the number of nodes of the Smolyak sparse grid $\mathcal{H}(N+k, N)=$ $\operatorname{dim}(A(N+k, N))$, the degrees-of-freedom of the complete polynomial space $W_{N}^{k}$ (2.13), and the tensor polynomial space $Z_{N}^{k}(2.14)$. (As shown in [42], the interpolation by $A(N+k, N)$ is exact in a polynomial space richer than $W_{N}^{k}$ but not as rich as $Z_{N}^{k}$.) It can be seen that as $N$ grows, the number of nodes from the Smolyak sparse grid is significantly less than $\operatorname{dim}\left(Z_{N}^{k}\right)$. Also, the relation (3.19) can be observed. This indicates that only at low dimensions, e.g., $N<5$, should the tensor product construction of interpolation nodes be considered.

The interpolation error in a space $F_{N}^{l}=\left\{f:[-1,1]^{N} \rightarrow \mathbb{R} \mid \partial^{|\mathbf{m}|} f\right.$ continues, $m_{i} \leq$ $l, \forall i\}$, where $\mathbf{m} \in \mathbb{N}_{0}^{N}$ and $\partial^{|\mathbf{m}|}$ is the usual $N$-variate partial derivative of order $|\mathbf{m}|$, 
is (cf. [3])

$$
\left\|\mathbb{I}_{N}-A(q, N)\right\|_{\infty} \leq C_{N, l} \cdot M^{-l} \cdot(\log M)^{(l+2)(N-1)+1},
$$

where $\mathbb{I}_{N}$ is the identity operator in a $N$-dimensional space and $M=\operatorname{dim}(\mathcal{H}(q, N)$ is the number of interpolation points.

Another intriguing property of the sparse grid constructed above is that it is also the nodes of a good cubature formula (3.11). Thus, we can efficiently evaluate the solution statistics in a similar way of (3.12). For analysis on integration error estimates of Smolyak sparse grid for multivariate integrals, see [41].

3.2.3. Stroud's cubature of degrees 2 and 3. For the $N$-hypercube spaces $[-1,1]^{N}$ considered in this paper, Stroud constructed a set of cubature points with $(N+1)$-point that is accurate for multiple integrals of polynomials of degree 2 , and a set of degree 3 with $2 N$-point [49]. The degree 2 formula, termed the Stroud-2 method hereafter, consists of points $\left\{y_{k}\right\}_{k=0}^{N}$ such that

$$
Y_{k}^{2 r-1}=\sqrt{\frac{2}{3}} \cos \frac{2 r k \pi}{N+1}, \quad Y_{k}^{2 r}=\sqrt{\frac{2}{3}} \sin \frac{2 r k \pi}{N+1}, \quad r=1,2, \ldots,[N / 2],
$$

where $[N / 2]$ is the greatest integer not exceeding $N / 2$, and if $N$ is odd $Y_{k}^{N}=$ $(-1)^{k} / \sqrt{3}$. The degree 3 formula, which will be called as Stroud-3 method, has the points $\left\{y_{k}\right\}_{k=1}^{2 N}$ such that

$$
Y_{k}^{2 r-1}=\sqrt{\frac{2}{3}} \cos \frac{(2 r-1) k \pi}{N}, \quad Y_{k}^{2 r}=\sqrt{\frac{2}{3}} \sin \frac{(2 r-1) k \pi}{N}, \quad r=1,2, \ldots,[N / 2],
$$

and if $N$ is odd $Y_{k}^{N}=(-1)^{k} / \sqrt{3}$.

It was proved in [38] that the Stroud-2 and -3 methods employ the minimal number of points for their corresponding integration accuracy. However, to the authors' knowledge, no theory exists on the approximation properties of the Lagrange interpolations constructed on these nodes. It is expected that the interpolation errors will be lower than degree 2 and 3 for the Stroud- 2 and -3 method, respectively.

In this paper, we will employ the Stroud- 2 and -3 nodes for stochastic collocation methods. For relatively low-order accuracy requirement in the solution statistics (expectations), the Stroud methods are highly efficient especially in high-dimensional spaces $(N \gg 1)$ because the numbers of nodal points are minimal. However, the accuracy cannot be improved any further and no error estimates can be obtained.

4. Applications to stochastic elliptic equations. In this section we use a stochastic elliptic problem to illustrate the applications of stochastic collocation methods. In particular, we consider for $\mathcal{P}$-a.e. $\omega \in \Omega$,

$$
\begin{aligned}
-\nabla \cdot(a(\omega, x) \nabla u(\omega, x)) & =f(\omega, x), & & \text { in } D, \\
u(\omega, x) & =0, & & \text { on } \partial D,
\end{aligned}
$$

where $a, f: \Omega \times D \rightarrow \mathbb{R}$ are known stochastic functions. Standard assumptions are made on the regularity of the input functions so that (4.1) is well-posed, e.g., $a \in L^{\infty}(\Omega, D)$ is strictly positive.

Again let us assume that the random input functions can be characterized by $N$ independent random variables $\left\{Y^{i}(\omega)\right\}_{i=1}^{N}$, whose images are bounded intervals 
and define an $N$-dimensional random space $\Gamma \subset \mathbb{R}^{N}$. Without loss of generality, let $\Gamma=[-1,1]^{N}$. Problem (4.1) can be then expressed in the strong form,

$$
\begin{aligned}
-\nabla \cdot(a(y, x) \nabla u(y, x)) & =f(y, x) & & \forall(y, x) \in \Gamma \times D, \\
u(y, x) & =0 & & \forall(y, x) \in \Gamma \times \partial D,
\end{aligned}
$$

and weak variational form: find $u \in L_{\rho}^{2}(\Gamma) \otimes H_{0}^{1}(D)$ such that

$$
\mathcal{K}_{\rho}(u, v ; \kappa)=\mathcal{I}_{\rho}(f, v) \quad \forall v \in L_{\rho}^{2}(\Gamma) \otimes H_{0}^{1}(D),
$$

where

$$
\mathcal{I}_{\rho}(v, w)=\int_{\Gamma} \rho(y) \int_{D} v(y, x) w(y, x) d x d y
$$

and

$$
\mathcal{K}_{\rho}(v, w ; \kappa)=\int_{\Gamma} \rho(y) \int_{D} a(y, x) \nabla v(y, x) \cdot \nabla w(y, x) d x d y .
$$

4.1. Formulations and error estimates. A stochastic collocation method, combined with a finite element method (FEM) in the physical space $D \subset \mathbb{R}^{d}$, takes the following form: for a given set of collocation nodes $\Theta_{N}=\left\{y_{k}\right\}_{k=1}^{M}$ on which the Lagrange interpolation is poised in an interpolation space $V_{I} \subset L_{\rho}^{2}(\Gamma)$, and a piecewise (continuous) FEM mesh $X_{d}^{h} \in H_{0}^{1}(D)$ (where $h>0$ is its mesh spacing parameter) that satisfies all the standard requirements on a FEM mesh, find as follows: for $k=1, \ldots, M, u_{k}^{h}(x) \equiv u^{h}\left(y_{k}, x\right) \in X_{d}^{h}$, such that

$$
\int_{D} a\left(y_{k}, x\right) \nabla u_{k}^{h}(x) \cdot \nabla \chi(x) d x=\int_{D} f\left(y_{k}, x\right) \chi(x) d x \quad \forall \chi \in X_{d}^{h} .
$$

The final numerical solution takes the form

$$
\hat{u}_{h}(y, x)=\sum_{k=1}^{M} u_{k}^{h}(x) L_{k}(y) .
$$

To study error estimates, let us define an error in term of the deterministic energy error, i.e.,

$$
\mathcal{E}_{G} \equiv\left(\mathbb{E} \int_{D} a\left(\nabla\left(u-\hat{u}_{h}\right)\right)^{2} d x\right)^{1 / 2}
$$

We can show that

$$
\begin{aligned}
\mathcal{E}_{G} & =\left(\int_{\Gamma} \rho(y) \int_{D} a(y, x)\left(\nabla\left(u-\hat{u}_{h}\right)\right)^{2} d x d y\right)^{1 / 2} \\
& \leq\left(\|\rho a\|_{L^{\infty}(\Gamma \times D)}\right)^{1 / 2} \min _{v \in V_{I} \otimes X_{h}^{d}}\|u-v\|_{L_{\rho}^{2}(\Gamma) \otimes H_{0}^{1}(D)} \\
& \leq\left(\|\rho a\|_{L^{\infty}(\Gamma \times D)}\right)^{1 / 2}\left(\mathcal{E}_{D}+\mathcal{E}_{\Gamma}\right),
\end{aligned}
$$

where $\mathcal{E}_{D}=\min _{v \in L_{\rho}^{2}(\Gamma) \otimes X_{h}^{d}}\|u-v\|_{L_{\rho}^{2}(\Gamma) \otimes H_{0}^{1}(D)}$ is the standard $H_{0}^{1}(D)$ FEM approximation error, and $\mathcal{E}_{\Gamma}$ is the $L_{\rho}^{2}(\Gamma)$ approximation error in the random space satisfying

$$
\mathcal{E}_{\Gamma}=\min _{v \in V_{I} \otimes H_{0}^{1}(D)}\|u-v\|_{L_{\rho}^{2}(\Gamma) \otimes H_{0}^{1}(D)} \leq\|u-\mathcal{I}(u)\|_{L_{\rho}^{2}(\Gamma) \otimes H_{0}^{1}(D)} \leq\|u-\mathcal{I}(u)\|_{\infty}=\mathcal{E}_{I} .
$$


The last term is the interpolation error, which can be bounded by the Lebesgue theorem (3.5). For the interpolation by Smolyak sparse grid, this error is (3.20). We hence have the following proposition.

Proposition 4.1 (separation of error estimates). The energy error defined in (4.6) can be effectively separated into two parts,

$$
\mathcal{E}_{G} \leq C\left(\mathcal{E}_{D}+\mathcal{E}_{I}\right)
$$

where $\mathcal{E}_{D}$ is the standard $H_{0}^{1}(D)$ FEM error in physical space $D, \mathcal{E}_{I}$ is the interpolation error in random space $\Gamma$, and $C$ is a constant independent of the discretizations in both $D$ and $\Gamma$.

4.2. Numerical results. Numerical implementations of stochastic Galerkin methods are not considered here as they have been considered in many earlier publications. For Galerkin methods in tensor polynomial spaces, see [12, 1, 2, 15]; in complete polynomial spaces, see $[19,20,21,52,54,55]$. Here we focus on numerical examples of stochastic collocation methods and consider the following problem in one spatial dimension $(d=1)$ and $N>1$ random dimensions:

$$
\frac{d}{d x}\left[a(y, x) \frac{d u}{d x}(y, x)\right]=0, \quad(y, x) \in \Gamma \times(0,1),
$$

with boundary conditions

$$
u(y, 0)=0, \quad u(y, 1)=1, \quad y \in \Gamma .
$$

The use of one-dimensional physical space $D$ allows us to focus on the properties of the stochastic collocation methods associated with the random space $\Gamma$. Extensions to two- and three-dimensional physical spaces are straightforward. We assume that the random diffusivity has the form

$$
a(y, x)=1+\sigma \sum_{k=1}^{N} \frac{1}{k^{2} \pi^{2}} \cos (2 \pi k x) Y^{k}(\omega),
$$

where $Y^{k}(\omega) \in[-1,1], k=1, \ldots, N$, are independent uniformly distributed random variables. The form of (4.11) is similar to those obtained from a Karhunen-Loève expansion with eigenvalues delaying as $1 / k^{4}$. Here we employ (4.11) to eliminate the errors associated with a numerical Karhunen-Loève solver and to keep the random diffusivity strictly positive as $N \rightarrow \infty$. The series in (4.11) converge as $N \rightarrow \infty$ and we have

$$
\mathbb{E}(a(y, x))=1, \quad 1-\frac{\sigma}{6}<a(y, x)<1+\frac{\sigma}{6} .
$$

The spatial discretization is done on a $p$-type spectral element with high-order modal expansions. The basis functions $\psi(\xi)$ in a standard interval $\xi \in(-1,1)$ are defined as

$$
\psi_{p}(\xi)= \begin{cases}\frac{1-\xi}{2}, & p=0, \\ \frac{1-\xi}{2} \frac{1+\xi}{2} P_{p-1}^{1,1}(\xi), & 0<p<P, \\ \frac{1+\xi}{2}, & p=P,\end{cases}
$$

where $P_{p}^{\alpha, \beta}(\xi)$ is the Jacobi polynomial of order $p$ with parameters $\alpha, \beta>-1$. For more details of such a basis, see [26]. In all the following computations, the spatial 

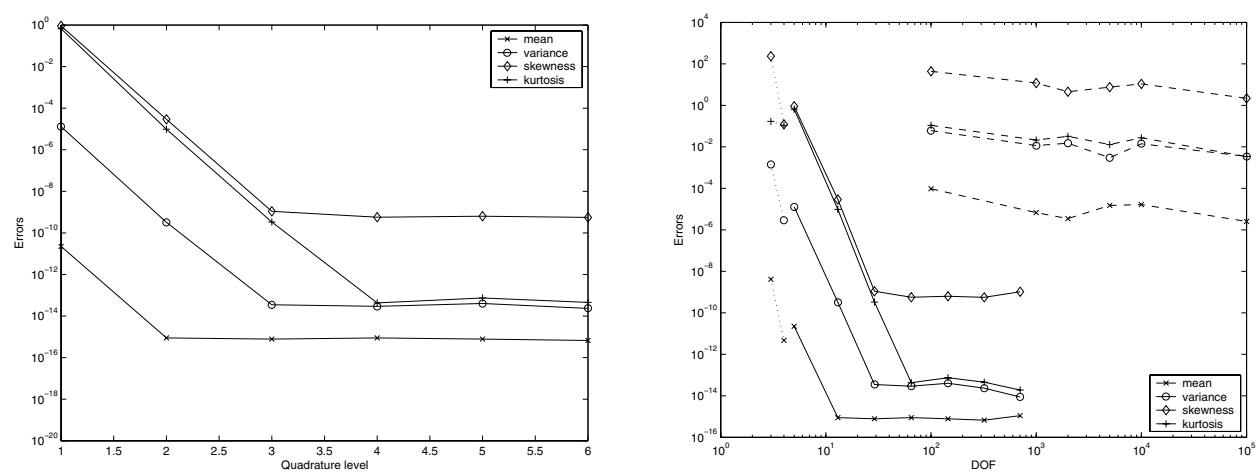

FIG. 4.1. Convergence of errors in moments with two-dimensional random input $(N=2)$. Left: convergence with respect to level of approximation $k$ of Smolyak formula $A(N+k, N)$; Right: convergence with respect to number of quadratures of different methods, Smolyak sparse grid (solid lines), Stroud's cubature (dotted lines), and Monte Carlo simulation (dashed lines).

discretizations are conducted with sufficiently high-order basis such that the spatial errors are subdominant.

The exact solution to this problem is

$$
u_{e}(y, x)=c_{1} \int \frac{1}{a(y, x)} d x+c_{2},
$$

where the two constants $c_{1}, c_{2}$ are determined by the boundary conditions. However, the closed form expression for the exact solution is not readily available.

4.2.1. Low-dimensional random inputs: Accuracy. In this section, a lowdimensional random input is assumed. In particular, we choose $N=2$. The "exact" solution is obtained by using the Smolyak sparse grid with level 8, i.e., $A(N+8, N)=$ $A(10,2)$.

In Figure 4.1, the errors in the first four moments of the solution are shown. On the left, the error of the Smolyak collocation method is shown. We observe that as the approximation level $k$ of $A(N+k, N)$ increases, the errors converge exponentially fast before reaching saturate levels.

On the right of Figure 4.1, the error convergence of a few different methods is shown with respect to the degree-of-freedom, i.e., the total number of interpolation points. It can be seen that the Smolyak method has the best accuracy; the Stroud2 and -3 methods approximate the lower moments (mean and variance) well, with considerable less degrees-of-freedom (only three and four points for the Stroud-2 and -3 method, respectively). On the other hand, the standard Monte Carlo method has the expected $O\left(\mathrm{DOF}^{-1 / 2}\right)$ convergence.

4.2.2. Moderate-dimensional random inputs. Here we choose a moderately high-dimensional random space with $N=8$. Figure 4.2 shows the error convergence with respect to degrees-of-freedom for different methods. Again, the Stroud-3 method offers solutions with accuracy comparable to the Smolyak method at level one $(k=1$ for $A(N+k, N)$ ), and with fewer nodes. In this case, both the Stroud method and Smolyak method are more efficient than Monte Carlo methods. We also observe that there is no clear sign of accuracy improvement in kurtosis, when comparing the Stroud-3 and Stroud-2 methods. (See also the results in Figure 4.1.) The reason is 


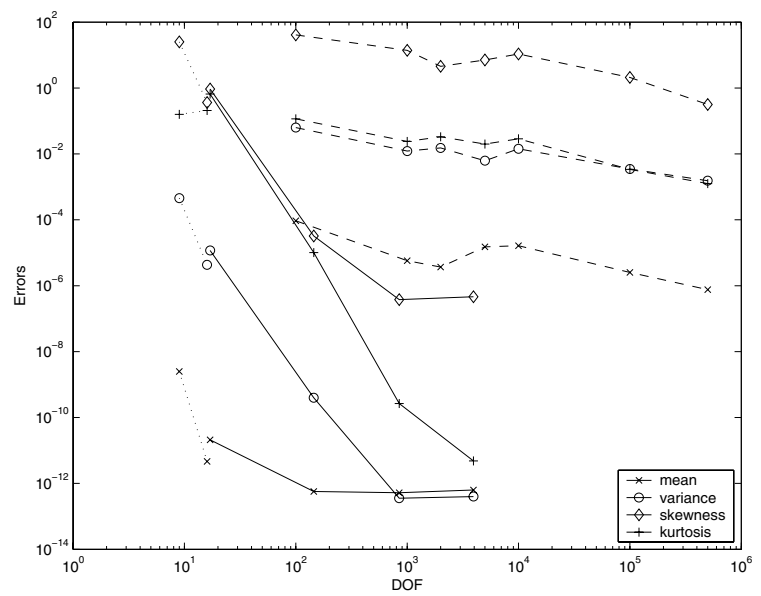

Fig. 4.2. Convergence of errors in moments with eight-dimensional random input $(N=8)$. Smolyak sparse grid $A(N+k, N), k \leq 4$ (solid lines), Stroud's cubature (dotted lines), and Monte Carlo simulation (dashed lines).
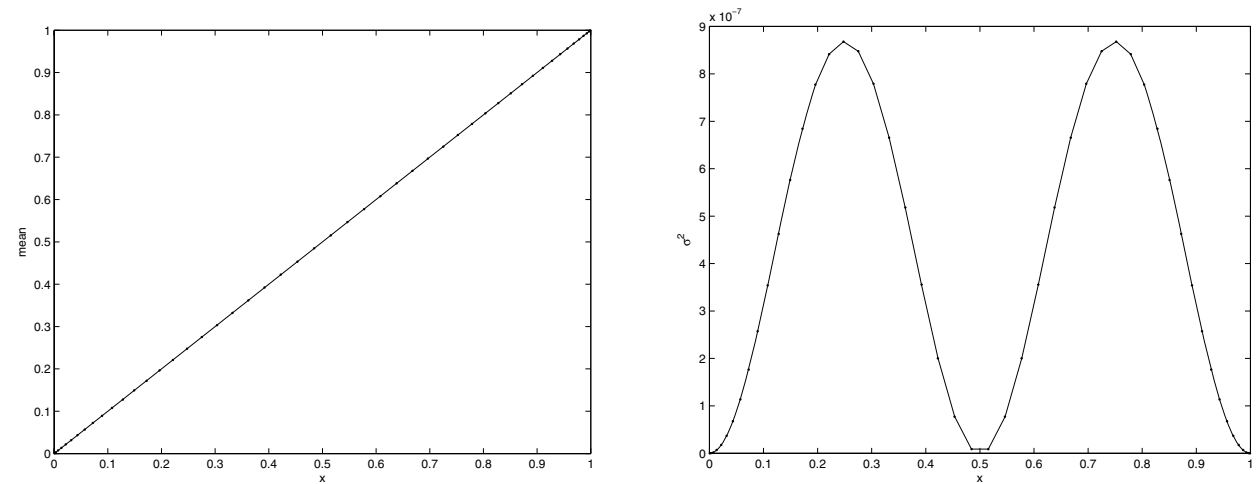

FIG. 4.3. Stochastic solution of (4.10) with 50-dimensional $(N=50)$ random input. Left: mean solution. Right: variance.

TABLE 4.1

Solutions at high-dimensional random input $(N=50)$, with Stroud-2 and -3 method, Smolyak method $A(N+k, N)$, and Monte Carlo method. Degrees-of-freedom for polynomial spaces: for $k=1$, $\operatorname{dim}\left(W_{N}^{k}\right)=51, \operatorname{dim}\left(Z_{N}^{k}\right) \approx 1.1 \times 10^{16} ;$ for $k=2, \operatorname{dim}\left(W_{N}^{k}\right)=1,326, \operatorname{dim}\left(Z_{N}^{k}\right) \approx 7.2 \times 10^{23}$.

\begin{tabular}{|c|c|c|}
\hline Methods & DOF & $\max \sigma_{u}^{2}(x)$ \\
\hline Stroud 2 & 51 & $8.67914(-7)$ \\
Stroud 3 & 100 & $8.67916(-7)$ \\
Smolyak $(k=1)$ & 101 & $8.67925(-7)$ \\
Smolyak $(k=2)$ & 5101 & $8.67919(-7)$ \\
MCS & 100 & $8.16677(-7)$ \\
MCS & 5000 & $8.73434(-7)$ \\
\hline
\end{tabular}

that kurtosis is the fourth moment of the solution and neither of the Stroud's methods is precise beyond third-order polynomials. 
4.2.3. High-dimensional random inputs: Efficiency. In this section, we focus on problem (4.10) with high-dimensional random input, $N=50$. In Figure 4.3, the solution profiles of mean and variance are shown. The maximums of variance are tabulated in Table 4.1. It can be seen that the Stroud-2, -3 method and Smolyak method with $k=2$ agree with each other well. With similar numbers of degrees-offreedom, Monte Carlo methods show noticeable differences. In this particular case, both the Stroud methods (Stroud-2 and Stroud-3) and the Smolyak method ( $k=1$ and $k=2$ ) are superior to the Monte Carlo method, and the Stroud-2 method is to be preferred, as it gives sufficiently accurate results (in variance) with only 51 nodes. The collocation points based on tensor products and Galerkin methods in tensor polynomial spaces $Z_{N}^{k}$ are clearly not practical, as $\operatorname{dim}\left(Z_{N}^{k}\right) \approx 7.2 \times 10^{23}$. The Galerkin methods in the complete polynomial spaces have number of expansion terms of 51 and 1,326 for $k=1,2$, respectively. The choice between a Galerkin method and a Smolyak collocation method in this case will depend on users, i.e., whether to use a deterministic solver more times or to design a solver for a coupled system of equations with less terms.

5. Time dependent problem: An illustrative example. Although the stochastic collocation algorithm is discussed in the context of boundary value problems, it is straightforward to apply it to time-dependent problems. Let us consider a damped motion of a particle in a bistable potential in the presence of a noisy periodic forcing. In particular, we consider for $\omega \in \Omega$

$$
\frac{d x}{d t}(t, \omega)=x-x^{3}+f(t, \omega), \quad x(0, \omega)=x_{0},
$$

where $f(t, \omega)$ is a $2 \pi$-periodic random process with zero mean value and a $2 \pi$-periodic covariance function, i.e., $\mathbb{E}[f(t, \omega)]=0$, and $\mathbb{E}\left[f\left(t_{1}, \omega\right) f\left(t_{2}, \omega\right)\right]=\sigma_{f}^{2} C_{f}\left(\left|t_{1}-t_{2}\right|\right)$, where $\sigma_{f}^{2}$ is the variance and $C_{f}(t)=C_{f}(t+2 \pi)$. We remark that with proper noisy input on top of a periodic driving force, the system (5.1) can exhibit complicated behavior such as stochastic resonance. (See, for example, [17].) However, the purpose of this example is on computational aspects of the algorithm, and we will restrict ourselves to cases with relatively simple dynamical behavior.

To model the periodic random process $f(t, \omega)$, we use a Fourier-type expansion

$$
\hat{f}(t, \omega)=\sum_{n=-K}^{K} f_{n}(\omega) e^{-i n t}
$$

for its characterization by a finite number of random variables. Here, the coefficients $f_{n}(\omega)=f_{n}^{r}(\omega)+i f_{n}^{i}(\omega)$ are complex random variables. It is straightforward to show that if $f_{n}^{r}$ and $f_{n}^{i}$ are statistically independent for all $n$ and have zero mean and variance of $C_{n} / 4$, where $C_{n}=\frac{1}{\pi} \int_{0}^{2 \pi} C_{f}(t) \cos (n t) d t$ are the coefficients of the Fourier cosine series of the $2 \pi$-periodic covariance function $C_{f}$, then the random field $\hat{f}(t, \omega)$ given by (5.2) approximates the prescribed $2 \pi$-periodic covariance function $C_{\hat{f}} \rightarrow C_{f}$ as $K \rightarrow \infty$. Let $y(\omega)=\left(f_{0}^{r}, f_{1}^{r}, f_{1}^{i}, \ldots, f_{K}^{r}, f_{K}^{i}\right)$, then $\hat{f}(t, \omega) \equiv \hat{f}(t, y(\omega))$ has $(2 K+1)$ random dimensions. (Note $f_{0}^{i}$ does not take effect in (5.2).) Obviously, $\hat{f}(t, y(\omega))$ is an approximation of $f(t, \omega)$, as (5.2) is a finite-term summation. In practice, we choose the number of expansion terms so that the contributions from the truncated terms are sufficiently small.

In this numerical example, we choose a $2 \pi$-periodic covariance function $C_{f}$ by extending the standard Gaussian covariance function $C_{G}(t)=\exp \left(-t^{2} / b^{2}\right)$ to the 


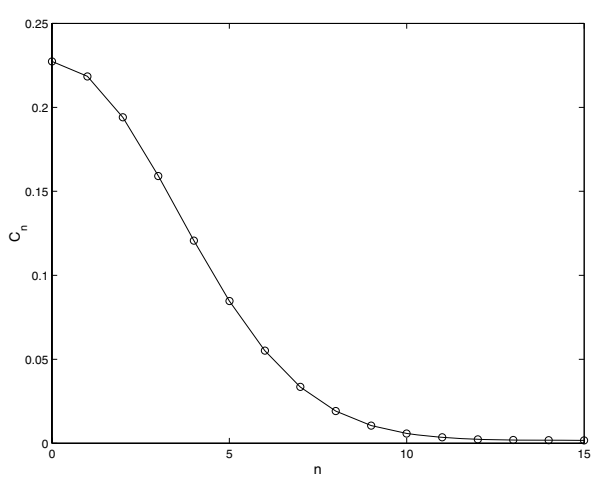

(a)

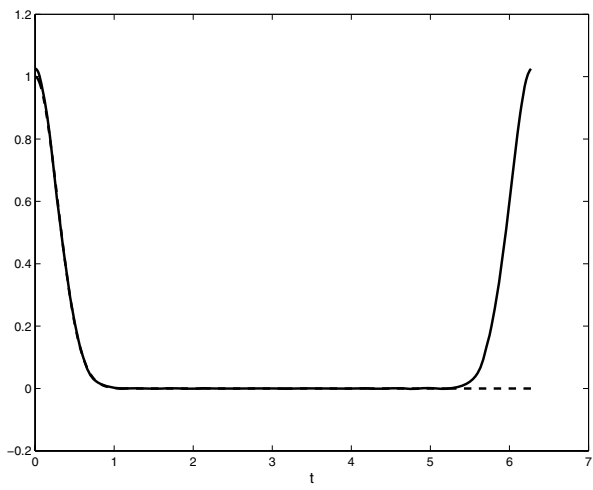

(b)

FIG. 5.1. Construction of a $2 \pi$-periodic covariance function $C_{f}$. (a) Decay of Fourier cosine coefficients $C_{n}, n=0,1, \ldots$; (b) reconstructed periodic covariance function (solid line) by extending the regular Gaussian covariance function (dashed line) $C_{G}(t)=\exp \left(t^{2} / b^{2}\right)$ with correlation length $b=0.4$.

periodic domain $(0,2 \pi)$, where $b$ is the correlation length. Hereafter we choose a moderately short correlation length $b=0.4$. In Figure 5.1(a), the decay of the Fourier cosine coefficient of $C_{f}$ is shown. Due to the relatively fast decay of these coefficients, we choose to use $K=10$ in the truncated series (5.2). Thus, $\hat{f}(t, y)$ has $N=2 K+1=$ 21 random dimensions. The constructed covariance function $C_{\hat{f}}(t)$ is shown in Figure 5.1(b), along with the standard nonperiodic Gaussian covariance function $C_{G}$, which we extended to the periodic domain.

Figure 5.2(a) shows four (arbitrarily chosen) realizations of $\hat{f}(t, y)$, constructed by $N=21(K=10)$ dimensions with correlation length $b=0.4$ and $\sigma=0.1$. Corresponding to these four realizations of forcing, the four realizations of solution to (5.1), with initial condition $x_{0}=0.5$, are shown in Figure 5.2(b). In this case, all realizations are attracted to one of the stable wells $(x=1)$ and oscillate nonlinearly around it. Subsequently, $\mathbb{E}[u(t)] \rightarrow 1$ and $\mathbb{E}\left[u^{2}(t)\right] \rightarrow \sigma_{u}^{2}=$ const. as $t \rightarrow \infty$. The computational results of the evolution of the standard deviation $\sigma_{u}(t)$ is shown in Figure 5.3. Stochastic collocation based on the Smolyak grid $A(N+k, N)$ is conducted for $k=1$ and $k=2$. With $N=21, A(N+1, N)$ has 43 points and $A(N+2, N)$ has 925 points. The results of standard deviation with $k=2$ shows little visual difference compared to $k=1$, and is regarded as the well-resolved solution. Results by Monte Carlo simulation are also shown in Figure 5.3. We observe the numerical oscillations resulted from the statistical errors, and the reduction of such errors from 100 realizations to 1,000 realizations is rather modest.

Problem (5.1) can also be solved via a stochastic Galerkin method. In the stochastic Galerkin method, we expand the solution as $u(t, y)=\sum_{i=1}^{M} u_{i}(t) \Phi_{i}(y)$, where $\left\{\Phi_{i}(y)\right\}$ is the multidimensional orthogonal basis defined in (2.13). The resulting equations for the expansion coefficients take the form

$$
\frac{d u_{m}}{d t}(t)=x_{m}-\frac{1}{\left\langle\Phi_{m}^{2}\right\rangle} \sum_{i=1}^{M} \sum_{j=1}^{M} \sum_{k=1}^{M} x_{i} x_{j} x_{k}\left\langle\Phi_{i} \Phi_{j} \Phi_{k} \Phi_{m}\right\rangle+f_{m}(t), \quad m=1, \ldots, M,
$$




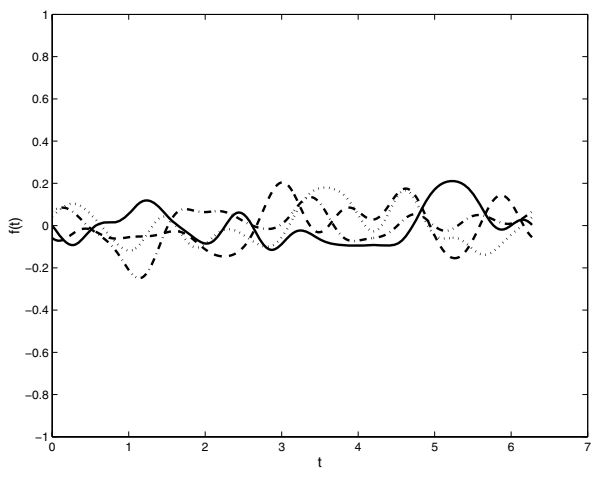

(a)

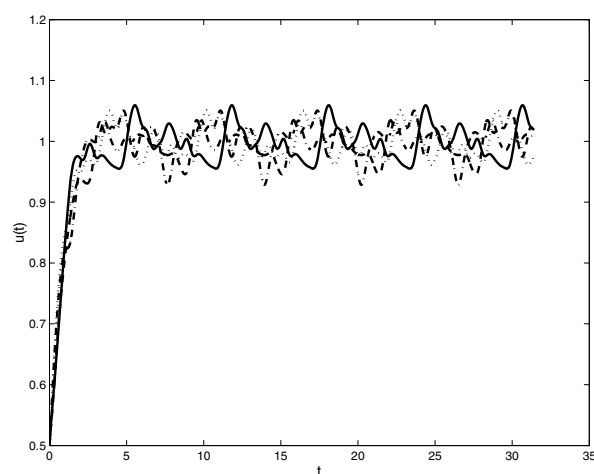

(b)

FIG. 5.2. Realizations of problem (5.1). (a) Four realizations of the periodic random forcing $\hat{f}(t, y(\omega))$; (b) four realizations of the solution $u(t, y(\omega))$ corresponding to the four realizations of forcing on the left.

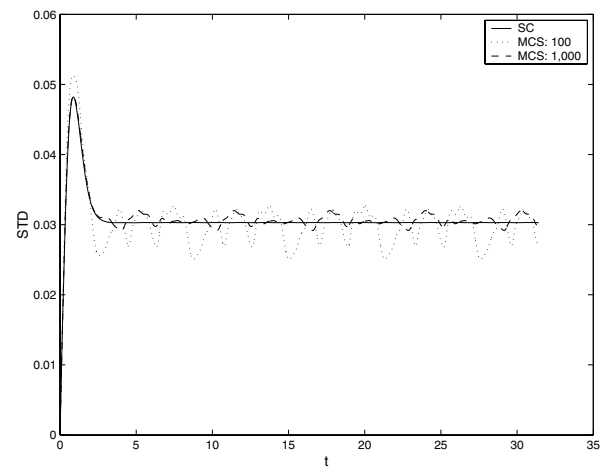

(a)

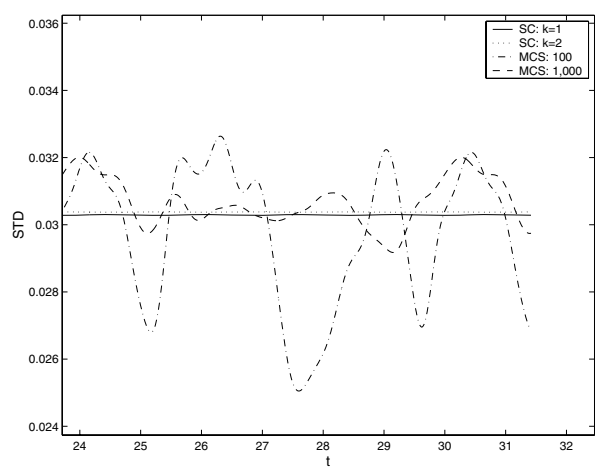

(b)

FIG. 5.3. Numerical results of the evolution of standard deviation (STD) of (5.1), computed by stochastic collocation (SC) and Monte Carlo simulations (MCS). (a) Solutions to $0 \leq t \leq 10 \pi$; (b) close-up view near $t \approx 10 \pi$.

where $\left\{f_{i}(t)\right\}$ are the expansion coefficients for the random forcing term. Solution of each of the $M$ number of equations requires a $M^{3}$ triple summations and storage (or on-the-fly evaluations) of the constants $\left\langle\Phi_{i} \Phi_{j} \Phi_{k} \Phi_{m}\right\rangle$ of the size $M^{4}$. In the case here, when $N=21, M=22$ for first-order expansion, and $M=252$ for second-order expansion. Neither the storage nor computing of these is negligible.

6. Summary. A class of high-order collocation methods is proposed in this paper. The method is a forge of several known mathematical and numerical components, e.g., sampling method, cubature formula/sparse grid, and collocational method. Its formulation is similar to that of a Monte Carlo method, where a deterministic equation is solved repetitively at each discrete point in the random space. However, in collocation methods the distribution of collocation points is fixed deterministically a priori and is determined through the aid of existing theory of multivariate polynomial inter- 
polation (or integration). Furthermore, by constructing the appropriate polynomial interpolations, stochastic collocation methods can achieve fast convergence (exponential convergence under sufficient smoothness conditions), similar to stochastic Galerkin methods. Thus, the stochastic collocation methods can be regarded as high-order "deterministic sampling methods." Also, when the interpolation error of certain collocation nodes is known, as in the case of the Smolyak sparse grid, we can obtain error estimates in strong form (e.g., the error estimate in (4.7)), whereas in cubature sampling or statistical sampling methods the interpolation errors are usually not known.

Several choices of the collocation nodal sets were investigated. It was found that the sparse grid based on Smolyak algorithm offers high accuracy and fast convergence, with a weak dependence on the number of random dimensions. An alternative, based on the Stroud-2 and -3 cubature, is optimal at lower orders. However, their accuracy is fixed and cannot be improved. Numerical experiments were conducted on an elliptic equation with random inputs, and we demonstrate that the error estimate can be effectively separated into two parts: one for the standard spatial discretization error and one for the interpolation error in random space. It was found that for random dimensions as high as 50, the stochastic collocation methods (including both Stroud's methods and Smolyak methods) are more efficient than brute-force Monte Carlo methods. With low accuracy, the Stroud-3 method is preferred to the Smolyak method with degree one $(k=1)$. On the other hand, the implementation of a collocation method is "embarrassingly" trivial, when compared to a stochastic Galerkin method which requires a specialized solver for the resulting coupled set of equations. However, the high-order collocation method based on Smolyak sparse grid has more degrees-offreedom than Galerkin methods in complete polynomial spaces. An example of a time dependent nonlinear ODE is also presented. The collocation method exhibits good convergence and accuracy for a 21-dimensional random input. This problem has a cubic nonlinearity, and a stochastic Galerkin approach, albeit with less expansion terms compared to the sparse grid collocation method, would incur nonnegligible extra computation cost due to the nonlinearity. The choice between a collocation method and a Galerkin method is problem dependent, and the advantage of collocation methods will be more noticeable for problems with more complicated forms of governing equations. A complete comparison between collocation and Galerkin methods depends on many factors, including detailed error estimates (particularly, in this paper we did not study the aliasing error by collocation methods) and assessment of the algorithm efficiency for a Galerkin method (for solving the coupled set of equations). This is our ongoing research.

\section{REFERENCES}

[1] I. BabušKa And P. Chatzipantelidis, On solving elliptic stochastic partial differential equations, Comput. Methods Appl. Mech. Engrg., 191 (2002), pp. 4093-4122.

[2] I. Babus̆Ka, R. Tempone, and G. Zouraris, Galerkin finite element approximations of stochastic elliptic differential equations, SIAM J. Numer. Anal., 42 (2004), pp. 800-825.

[3] V. Barthelmann, E. Novak, And K. Ritter, High dimensional polynomial interpolation on sparse grid, Adv. Comput. Math., 12 (1999), pp. 273-288.

[4] I. Berezin And N. Zhidkov, Computing Methods, Addison-Wesley, Reading, MA, 1965.

[5] C. Bernardi and Y. Maday, Properties of some weighted Sobolev spaces and application to spectral approximations, SIAM J. Numer. Anal., 26 (1989), pp. 769-829.

[6] C. Canuto and A. Quarteroni, Approximation results for orthogonal polynomials in Sobolev spaces, Math. Comput., 38 (1982), pp. 67-86.

[7] Q.-Y. Chen, D. Gottlieb, And J. Hesthaven, Uncertainty analysis for the steady-state flows in a dual throat nozzle, J. Comput. Phys., 204 (2005), pp. 387-398. 
[8] K. Chung And T. YaO, On lattices admitting unique Lagrange interpolations, SIAM J. Numer. Anal., 14 (1977), pp. 735-743.

[9] R. Cools, Monomial cubature rules since 'Stroud': A compilation, part 2, J. Comput. Appl. Math., 112 (1999), pp. 21-27.

[10] R. Cools and P. Rabinowitz, Monomial cubature rules since 'Stroud': A compilation, J. Comput. Appl. Math., 48 (1993), pp. 309-326.

[11] P. DAVIS, Interpolation and Approximation, Dover, New York, 1975.

[12] M. Deb, I. BABušKA, AND J. Oden, Solution of stochastic partial differential equations using Galerkin finite element techniques, Comput. Methods Appl. Mech. Engrg., 190 (2001), pp. 6359-6372.

[13] G. Fishman, Monte Carlo: Concepts, Algorithms, and Applications, Springer-Verlag, New York, 1996.

[14] B. Fox, Strategies for Quasi-Monte Carlo, Kluwer, Dordrect, The Netherlands, 1999.

[15] P. Frauenfelder, C. Schwab, and R. Todor, Finite elements for elliptic problems with stochastic coefficients, Comput. Methods Appl. Mech. Engrg., 194 (2005), pp. 205-228.

[16] D. Gamerman, Markov Chain Monte Carlo: Stochastic Simulation for Bayesian Inference, Chapman \& Hall, London, 1997.

[17] L. Gammaitoni, P. Hanggi, P. Jung, and F. Marchesoni, Stochastic resonance, Rev. Modern Phys., 70 (1998), pp. 223-287.

[18] C. Gardiner, Handbook of Stochastic Methods: For Physics, Chemistry and the Natural Sciences, 2nd ed., Springer-Verlag, New York, 1985.

[19] R. Ghanem, Ingredients for a general purpose stochastic finite element formulation, Comput. Methods Appl. Mech. Engrg., 168 (1999), pp. 19-34.

[20] R. Ghanem, Stochastic finite elements for heterogeneous media with multiple random nonGaussian properties, ASCE J. Engrg. Mech., 125 (1999), pp. 26-40.

[21] R. Ghanem and P. Spanos, Stochastic Finite Elements: A Spectral Approach, Springer-Verlag, New York, 1991.

[22] W. Gilks, S. Richardson, And D. Spiegelhalter, Markov Chain Monte Carlo in Practice, Chapman \& Hall, London, 1995.

[23] M. Grigoriu, Simulation of stationary non-Gaussian translation processes, J. Engrg. Mech., 124 (1998), pp. 121-126.

[24] E. Isaacson and H. Keller, Analysis of Numerical Methods, John Wiley, New York, 1966.

[25] I. Karatzas And S. Shreve, Brownian Motion and Stochastic Calculus, Springer-Verlag, New York, 1988.

[26] G. Karniadakis and S. Sherwin, Spectral/hp Element Methods for CFD, Oxford University Press, London, 1999.

[27] M. Kleiber And T. HiEn, The stochastic finite element method, John Wiley, New York, 1992.

[28] P. Kloeden and E. Platen, Numerical Solution of Stochastic Differential Equations, Springer-Verlag, New York, 1999.

[29] O. Le Maitre, O. Knio, H. Najm, and R. Ghanem, Uncertainty propagation using WienerHaar expansions, J. Comput. Phys., 197 (2004), pp. 28-57.

[30] O. Le Maitre, H. Najm, R. Ghanem, and O. Knio, Multi-resolution analysis of Wiener-type uncertainty propagation schemes, J. Comput. Phys., 197 (2004), pp. 502-531.

[31] W. Liu, T. Belytschko, and A. Mani, Probabilistic finite elements for nonlinear structural dynamics, Comput. Methods Appl. Mech. Engrg., 56 (1986), pp. 61-81.

[32] W. Liu, T. Belytschko, and A. Mani, Random field finite elements, Internat. J. Numer. Methods Engrg., 23 (1986), pp. 1831-1845.

[33] M. LoÈve, Probability Theory, 4th ed., Springer-Verlag, New York, 1977.

[34] W. LoH, On Latin hypercube sampling, Ann. Statist., 24 (1996), pp. 2058-2080.

[35] G. Lorentz, Approximation of Functions, Holt, Rinehart and Winston, New York, 1966.

[36] N. Madras, Lectures on Monte Carlo Methods, American Mathematical Society, Providence, RI, 2002.

[37] L. Mathelin And M. Hussaini, A stochastic collocation algorithm for uncertainty analysis, Tech. Report NASA/CR-2003-212153, NASA Langley Research Center, 2003.

[38] I. Mysovskin, Proof of the minimality of the number of nodes in the cubature formula for a hypersphere, USSR Comput. Math. Math. Phys., 6 (1966), pp. 15-27.

[39] H. Niederreiter, Random Number Generation and Quasi-Monte Carlo Methods, SIAM, Philadelphia, 1992.

[40] H. Niederreiter, P. Hellekalek, G. Larcher, and P. Zinterhof, Monte Carlo and QuasiMonte Carlo Methods 1996, Springer-Verlag, New York, 1998.

[41] E. NovaK AND K. RitTER, High dimensional integration of smooth functions over cubes, Numer. Math., 75 (1996), pp. 79-97.

[42] E. NovaK AND K. RitTeR, Simple cubature formulas with high polynomial exactness, Con- 
structive Approx., 15 (1999), pp. 499-522.

[43] B. Oksendal, Stochastic Differential Equations. An Introduction with Applications, 5th ed., Springer-Verlag, New York, 1998.

[44] M. Rosenblatt, Remark on a multivariate transformation, Ann. Math. Statist., 23 (1953), pp. $470-472$.

[45] S. Sakamoto And R. Ghanem, Simulation of multi-dimensional non-Gaussian non-stationary random fields, Probab. Engrg. Mech., 17 (2002), pp. 167-176.

[46] S. SMOLYAK, Quadrature and interpolation formulas for tensor products of certain classes of functions, Soviet Math. Dokl., 4 (1963), pp. 240-243.

[47] C. Solze And R. Ghanem, Physical systems with random uncertainties: Chaos representations with arbitrary probability measure, SIAM. J. Sci. Comput., 26 (2004), pp. 395-410.

[48] M. Stein, Large sample properties of simulations using Latin hypercube sampling, Technometrics, 29 (1987), pp. 143-151.

[49] A. Stroud, Remarks on the disposition of points in numerical integration formulas, Math. Comput., 11 (1957), pp. 257-261.

[50] G. Wasilkowski and H. Wozniakowski, Explicit cost bounds of algorithms for multivariate tensor product problems, J. Complexity, 11 (1995), pp. 1-56.

[51] N. Wiener, The homogeneous chaos, Amer. J. Math., 60 (1938), pp. 897-936.

[52] D. XIU AND G. KARNIADAKIS, Modeling uncertainty in steady state diffusion problems via generalized polynomial chaos, Comput. Methods Appl. Math. Engrg., 191 (2002), pp. 49274948.

[53] D. Xiu and G. Karniadakis, The Wiener-Askey polynomial chaos for stochastic differential equations, SIAM J. Sci. Comput., 24 (2002), pp. 619-644.

[54] D. XIU And G. KARNiAdakis, Modeling uncertainty in flow simulations via generalized polynomial chaos, J. Comput. Phys., 187 (2003), pp. 137-167.

[55] D. XiU And G. Karniadakis, A new stochastic approach to transient heat conduction modeling with uncertainty, Internat. J. Heat Mass Trans., 46 (2003), pp. 4681-4693.

[56] D. XiU and G. Karniadakis, Supersensitivity due to uncertain boundary conditions, Internat. J. Numer. Meth. Engrg., 61 (2004), pp. 2114-2138.

[57] D. XIU AND S. Sherwin, Uncertainty modelling in hyperbolic systems and its applications in reduced modelling of a human arterial network, in Proceedings of International Conference of High-Order and Spectral Methods (ICOSAHOM), Providence, RI, 2004.

[58] F. Yamazaki and M. Shinozuka, Digital generation of non-Gaussian stochastic fields, J. Engrg. Mech., 114 (1988), pp. 1183-1197. 